\title{
SENSITIVITY ANALYSIS OF FLUTTER RESPONSE OF A WING INCORPORATING FINITE-SPAN CORRECTIONS
}

\author{
Jason C. Issac \\ Rakesh K. Kapania \\ and \\ J.-F. M. Barthelemy
}

Extended abstract submitted to the 5th AIAA/NASA/USAF/ISSMO Symposium on Multidisciplinary Analysis and Optimization, Panama City, Florida, September 7-9, 1994.

Report on "Shape Sensitivity Analysis of Static and Dynamic Aeroelastic Responses" (NAG1-1411) 


\title{
Sensitivity Analysis of Flutter Response of a Wing Incorporating Finite-Span Corrections
}

\author{
Jason Cherian Issac* and Rakesh K. Kapania ${ }^{\dagger}$ \\ Virginia Polytechnic Institute and State University \\ Blacksburg, Virginia 24061-0203 \\ and \\ Jean-Francois M. Barthelemy \\ NASA, Langley Research Center \\ Hampton, VA 23681
}

\begin{abstract}
Flutter analysis of a wing is performed in compressible flow using state-space representation of the unsteady aerodynamic behavior. Three different expressions are used to incorporate corrections due to the finite-span effects of the wing in estimating the lift-curve slope. The structural formulation is based on a Rayleigh-Ritz technique with Chebyshev polynomials used for the wing deflections. The aeroelastic equations are solved as an eigenvalue problem to determine the flutter speed of the wing. The flutter speeds are found to be higher in these cases, when compared to that obtained without accounting for the finite-span effects. The derivatives of the flutter speed with respect to the shape parameters, namely, (i) aspect ratio, (ii) area, (iii) taper ratio and (iv) sweep angle, are calculated analytically. The shape sensitivity derivatives give a linear approximation to the flutter speed curves over a range of values of the shape parameter which is perturbed. Flutter and sensitivity calculations are performed on a wing using a lifting-surface unsteady aerodynamic theory using modules from a system of programs called FAST.

* Graduate Research Assistant, Aerospace and Ocean Engineering, Student Member AIAA.

$\dagger$ Associate Professor, Aerospace and Ocean Engineering, Associate Fellow AIAA.

$\ddagger$ Senior Aerospace Engineer, Interdisciplinary Research Office, Structural Dynamics Division, Senior Member AIAA.
\end{abstract}




\section{Introduction}

Several unsteady aerodynamic codes exist and new codes are emerging for application to numerous aeroelastic problems. These codes essentially differ in the prediction of the lift forces and moments acting on a wing. The structural and aerodynamic characteristics of the wing are functions of its shape parameters and hence the flutter response is sensitive to changes in shape. Sensitivity analysis is an important tool which yields information about the dependence of the aeroelastic instability on the design parameters of the wing.

In recent years, considerable efforts are being made to integrate the aerodynamic, structural and control aspects of the design of an aircraft. It is desirable that the unsteady aerodynamic airloads be expressed in a state-space form. Leishman and $\mathrm{Nguyen}{ }^{1}$ have represented the aerodynamic indicial response functions for compressible flow by up to three-pole approximations, the response consisting of two parts, one due to non-circulatory loading and the other due to circulatory loading. Using this approach, the aeroelastic equations can be written as a set of first-order ordinary differential equations as given by Leishman and Crouse ${ }^{2}$. This has advantages over the CFD-based methods in the sense that the CFD methods are in general computationally very expensive.

The problem of flutter instability was studied by Kapania and Issac ${ }^{3}$ using the statespace aerodynamic representation by Leishman and Nguyen ${ }^{1}$. However, in this study, a constant value of the section lift-curve slope was used. But for a $3 \mathrm{D}$ finite wing, the wing lift-curve slope depends on the planform of the wing and these finite-span effects had been neglected in this study.

The planform parameters of the wing play an important role in its lifting characteristics. It is observed that neglecting the finite-span effects of a wing has the effect of overpredicting the lift forces and moments acting on the wing. In other words, if an aerodynamic theory with an infinite wing lift-curve slope is used in an aeroelastic analysis, it could result in flutter speeds which are more conservative. Several approximations for the lift-curve slope have been reported in literature which take into account the finite-span corrections for a finite aspect ratio wing. 
Diederich ${ }^{4}$ derived approximate expressions for the wing lift-curve slope based on both lifting-line and lifting-surface theories. A planform parameter was introduced which is a function of the 2D lift-curve slope, aspect ratio and sweep angle to correlate the aerodynamic characteristics of the wing. Hauptman and $\mathrm{Miloh}^{5}$ developed an analytical solution for the lifting surface problem of an elliptic wing. Simple explicit expressions were derived for the lift and moment coefficients in terms of the aspect ratio. Laitone ${ }^{6}$ compares a set of approximate solutions for the finite wing lift-curve slope with exact solutions derived for an elliptic planform flat plate. These approximations to the lift-curve slope based on planform parameters of the wing, thus enables to perform an aeroelastic analysis which yields a better estimate of the flutter speed.

Sensitivity analysis is becoming an important design tool in engineering design applications. Sensitivity derivatives are of great importance in multidisciplinary design optimization of aircrafts. Sobieski ${ }^{7}$ discusses in detail the System Design Derivatives which help in understanding the effect a particular design variable would have on the desired performance of the system, if it were perturbed by a small percentage from its original value.

The sensitivity derivatives of a system can be found using either analytical or finite difference methods. Analytical sensitivity analysis has found increased interest in engineering design as it eliminates uncertainity in the choice of step size needed in the finite difference method. The step size if too large leads to truncation errors and if too small leads to round-off errors. For example, recently Livne ${ }^{8}$ observed that as higher order polynomials are used in the Ritz functions for better modeling of the structure, the more sensitive is the finite difference derivative to the step-size used and in some cases, it is impossible to obtain any valuable information by finite differences.

Rudisill and $\mathrm{Bhatia}^{9}$ developed expressions for the analytical derivatives of the eigenvalues, reduced frequency and flutter speed with respect to structural parameters for use in minimizing the total mass. Pedersen and Seyranian ${ }^{10}$ examined the change in flutter load as a function of change in stiffness, mass, boundary conditions or load distribution. They showed how sensitivity analysis can be performed without any new eigenvalue analysis. 
The solution to the main and an adjoint problem provide all the necessary information for evaluating sensitivities.

Hawk and Bristow ${ }^{11}$ developed aerodynamic sensitivity analysis capabilities in subcritical compressible flow. They first analyzed a baseline configuration, and then calculated a matrix containing partial derivatives of the potential at each control point with respect to each known geometric parameter by applying a first order expansion to the baseline configuration. The matrix of partial derivatives is used in each iteration cycle to analyze the perturbed geometry.

Barthelemy and Bergen ${ }^{12}$ explored the analytical shape sensitivity derivatives of the wing's aeroelastic characteristics, such as section lift, angle of attack, rolling moment, induced drag and divergence dynamic pressure, for subsonic subcritical flow, with respect to geometric parameters. Results showed the characteristics nonlinearity to be small enough to be well approximated by sensitivity based linear approximations. These approximations are valid within a range that is useful to designers in the initial design phase. Kapania ${ }^{13}$ has obtained sensitivity derivatives of the flutter speed of a two dimensional airfoil in incompressible flow with respect to the mass and stiffness parameters. Kapania, Bergen and Barthelemy ${ }^{14}$ have obtained the shape sensitivity derivatives of the flutter response of a laminated wing in incompressible flow.

In this paper, the aerodynamic state-space model ${ }^{1}$ is used to represent the unsteady aerodynamic airloads on a wing. Three different expressions are used to incorporate corrections due to the finite-span effects of the wing in estimating the lift-curve slope. The structural formulation is based on a Rayleigh-Ritz technique with Chebyshev polynomials chosen for the displacement function. The aeroelastic equations for the wing are solved as an eigenvalue problem to determine the stability. The derivatives of the flutter speed are calculated with respect to the shape parameters, namely (i) aspect ratio, (ii) area, (iii) taper ratio, and (iv) sweep analytically. Flutter speed and sensitivity calculations are also performed on a wing using lifting-surface unsteady aerodynamics using modules from a system of programs called FAST (Flutter Analysis System). Flutter speeds are obtained using a $\mathrm{V}$-g type of solution. 


\section{Aeroelastic analysis of the wing}

The aerodynamic and structural model used for the flutter analysis are described in detail in Ref. 3. Using the state-space unsteady aerodynamic representation by Leishman and Nguyen $^{1}$, the aeroelastic equations of the wing are written as a set of first order ordinary differential equations. The system of equations is solved as an eigenvalue problem to determine the stability.

In the study performed in Ref. 3, the finite-span effects of the wing were neglected. However, for a finite aspect ratio wing, the wing lift-curve slope is found to be a function of the planform parameters and corrections to lift-curve slope due to the finite-span effects have to be accounted for.

\section{Corrections due to finite-span effects}

The finite-span effects on the aerodynamic characteristics of a wing are found to be functions of the planform parameters. A number of approximations have been reported in literature which accounts for the corrections to a finite aspect ratio wing.

Correction 1: One of the representations for the lift-curve slope of a finite wing is given by Hauptman and Miloh ${ }^{5}$, which for compressible flow can be written as

$$
C_{N_{\alpha}}=\frac{4}{\left[k+\frac{E^{2}(h)}{k+(\arcsin h) / h}\right]} \frac{1}{\sqrt{1-M^{2} \cos ^{2} \Lambda}}
$$

where $k=4 / \pi A R$ and $h=\sqrt{\left(1-k^{2}\right)}$ and $A R$ is the aspect ratio of the wing. $E(h)$ is the complete elliptic integral of the second kind and is given by

$$
E(h)=\int_{0}^{\pi / 2}\left(1-h^{2} \sin ^{2} \phi\right)^{1 / 2} d \phi
$$

Correction 2: Another approximation ${ }^{6}$ to the lift-curve slope for a finite aspect ratio wing can be written for compressible flow as

$$
C_{N_{\alpha}}=\left[\frac{2 \pi}{1+\left(\frac{2}{A R}\right)(1+\tau)}\right] \frac{1}{\sqrt{1-M^{2} \cos ^{2} A}}
$$


where $A R$ is the aspect ratio and $A$ is the sweep angle. Kida and $M$ Miyai ${ }^{15}$ have shown that for wings with $A R \geq 4 / \pi$, the correction factor $\tau$ can be written as

$$
\tau=\left(\frac{8}{\pi^{2} A R}\right)[\ln (\pi A R)-1]+O\left(\frac{1}{A R^{2}}\right)
$$

Correction 3: Laitone in Ref. 6 reports of a very remarkable approximation derived by Helmbold ${ }^{16}$ for the lift-curve slope of a finite wing, which for compressible flow can be written as

$$
C_{N_{\alpha}}=\left[\frac{2 \pi A R}{2+\left(4+A R^{2}\right)^{1 / 2}}\right] \frac{1}{\sqrt{1-M^{2} \cos ^{2} 1}}
$$

where $A R$ is the aspect ratio and $A$ is the sweep angle.

All the three expressions for the lift-curve slope are used to compute the flutter speed of the wing. The results obtained are compared with the flutter speed calculated by using a value of $C_{N_{\alpha}}(M)$, which are experimental values of $C_{N_{\alpha}}$ obtained as functions of Mach numbers for an airfoil section.

\section{Sensitivity Analysis}

The aeroelastic equations obtained as a set of first order ODEs is of the form

$$
[P] \dot{\mathbf{w}}=[Q] \mathbf{w}
$$

which could be written as

$$
\dot{\mathbf{w}}=[E] \mathbf{w}
$$

where $[E]=[P]^{-1}[Q]$

The derivative of the $i$ th eigenvalue with respect to the critical speed is given by

$$
\frac{\partial \lambda^{i}}{\partial V_{f}}=\frac{\left\{e_{l}^{i}\right\}^{T} \frac{\partial[E]}{\partial V_{f}}\left\{e_{r}^{i}\right\}}{\left\{e_{l}^{i}\right\}^{T}\left\{e_{r}^{i}\right\}}
$$

where $\left\{e_{l}^{i}\right\}$ and $\left\{e_{r}^{i}\right\}$ are the $i$ th left and right eigenvectors respectively.

Similarly, the derivative of the $i$ th eigenvalue with respect to any parameter $p$ is given by

$$
\frac{\partial \lambda^{i}}{\partial p}=\frac{\left\{e_{l}^{i}\right\}^{T} \frac{\partial[E]}{\partial p}\left\{e_{r}^{i}\right\}}{\left\{e_{l}^{i}\right\}^{T}\left\{e_{r}^{i}\right\}}
$$


$\frac{\partial[E]}{\partial p}$ can be conveniently written as

$$
\frac{\partial[E]}{\partial p}=\frac{\partial[P]^{-1}}{\partial p}[Q]+[P]^{-1} \frac{\partial[Q]}{\partial p}
$$

and can be computed analytically, where

$$
\frac{\partial[P]^{-1}}{\partial p}=-[P]^{-1} \frac{\partial[P]}{\partial p}[P]^{-1}
$$

The analytical derivative of the critical speed with respect to parameter $p$ is then given by

$$
\frac{\partial V_{f}}{\partial p}=-\frac{\operatorname{Real}\left(\frac{\partial \lambda^{i}}{\partial p}\right)}{\operatorname{Real}\left(\frac{\partial \lambda^{i}}{\partial V_{f}}\right)}
$$

The $[E]$ matrix is composed of mass, stiffness and aerodynamic matrices. The derivatives of the elements in the matrix are obtained by taking the analytical derivatives of those terms that are explicit functions of the shape parameters which are given in Ref. 17 . It should be noted that, with the corrections applied to include the finite-span effects, the wing lift-curve slope is sensitive to changes in shape.

\section{Evaluation Analysis}

Flutter calculations for a wing in transonic flow was performed in Ref. 3. Subsequently sensitivity analysis of the flutter speed of the wing with respect to shape parameters was carried out. The wing is shown in Fig. 1. The wing skins are made of $0^{\circ}$ laminated Graphite/Epoxy (T300/N5208) with the following material properties: $E_{1}=181 \times 10^{9} P a$, $E_{2}=10.3 \times 10^{9} \mathrm{~Pa}, \nu_{12}=0.28, G_{12}=7.17 \times 10^{9} \mathrm{~Pa}$ and $\rho=1600 \mathrm{~kg} / \mathrm{m}^{3}$. In these calculations performed, the finite-span effects of the wing were neglected.

Three different expressions are used in this study to incorporate the corrections due to the finite-span effects in estimating the lift-curve slope of the wing. With this correction applied to the aerodynamics code, the wing was examined for aeroelastic instability. The flutter speed of the wing, as predicted by these three corrections to the lift-curve slope and the $2 \mathrm{D}$ lift-curve slope are shown in Fig. 2 as a function of the quarter-chord sweep angle. 
The difference between the flutter speeds obtained using the three corrections is too small, for this wing, as seen from Fig. 2. It can be seen that when 2D lift-curve slope is used, the flutter results are more conservative.

Table 1 gives the flutter speed predicted, with and without accounting for the finitespan effects of the wing. The results obtained by using three different expressions for the wing lift-curve slope are shown. For the high aspect ratio wing analysed, it can be seen that the three expressions for finite wing lift-curve slope give almost the same flutter speeds, but the speeds are higher than that predicted by using the 2D lift-curve slope. The sensitivity derivative of the flutter speed with respect to shape parameters, obtained analytically, are given in Table 2.

The flutter speeds of the wing obtained by perturbing one shape parameter at a time from the baseline configuration are shown in Fig. 3-6. The flutter prediction from the different corrections applied are plotted here. Similar curves for the flutter speed obtained without applying finite-span corrections were plotted in Ref. 3. The analytical sensitivity calculation is also superposed. The sensitivity derivative obtained forms a tangent to the flutter speed curve at the value of the shape parameter at which it is computed.

By performing one sensitivity calculation at the baseline analytically, this method gives a linear approximation to the flutter speeds of the wing for changes in the wing shape parameters about the baseline. This information is useful for preliminary design purposes, as it avoids the necessity of a reanalysis for small changes in any of the shape parameters.

\section{Work in progress}

We are working on flutter analysis of a wing using a lifting-surface unsteady aerodynamic theory which leads to a more accurate flutter solution. The modules from a system of programs called FAST ${ }^{18}$ (Flutter Analysis System) are used to obtain the generalized aerodynamic forces on the wing. A free vibration analysis of the wing is performed and the vibration modes from this analysis are fed into the modes processing module from FAST. The subsonic kernel function matrix program then solves the subsonic downwash integral equation for the oscillating planar wing lifting surface. The generalized force module from 
FAST then computes the aerodynamic forces from the subsonic kernel matrices. The flutter speed of the wing is obtained using a $\mathrm{V}-\mathrm{g}$ type of solution. Sensitivity calculations are performed on the flutter speed of the wing with respect to the shape parameters. Studies on flutter and sensitivity calculations on the HSCT wing are performed using these methods.

\section{Concluding Remarks}

The compressible unsteady aerodynamic theory using indicial response functions is used to represent the aerodynamic forces and moments on a finite span wing. Three different expressions are used in this study to incorporate the finite-span corrections in estimating the lift-curve slope of the wing. Using this aerodynamic state-space model and the structural formulation based on Ritz technique, aeroelastic analysis of wings were carried out. It is found that incorporating the finite-span corrections, gives higher flutter speeds for the wing, as compared to using the 2D lift-curve slope. The use of Chebyshev polynomials for Ritz functions gives the added benefit of closed form analytical expressions for the derivatives of stiffness and mass matrices with respect to the shape design parameters of the wing. The shape sensitivity derivatives of the flutter speed of the wing were computed analytically. Flutter speed and sensitivity calculations are also performed using a lifting-surface unsteady aerodynamic theory using the generalized aerodynamic forces from a system of programs called FAST and a V-g type of solution. These shape derivatives of the flutter response of a wing would be very useful to a designer in the initial design phase, thus avoiding the necessity of a reanalysis for small changes in the design parameters.

\section{Acknowledgement}

The work presented here is a part of the work done in the project sponsored by NASA, Langley Research Center under a grant NAG-1-1411 to VPI\&SU. 


\section{References}

1 Leishman, J.G., and Nguyen, K.Q., "State-Space Representation of Unsteady Airfoil Behavior," AIAA Journal, Vol. 28, No. 5, May 1990, pp. 836-844.

2 Leishman, J.G., and Crouse, G.L., "Transonic Aeroelasticity Analysis Using StateSpace Unsteady Aerodynamic Modelling," Journal of Aircraft, Vol. 29, No. 1, 1992, pp. $153-160$.

3 Kapania, R.K., and Issac, J.C., "Sensitivity Analysis of Aeroelastic Response of a Wing in Transonic Flow," AIAA Journal, Accepted for publication. See also "Sensitivity Analysis of Flutter Response of a Typical Section and a Wing in Transonic Flow," presented at the 34th AIAA/ASME/ASCE/AHS/ASC Structures, Structural Dynamics and Materials Conference, La Jolla, CA, 1993.

4 Diederich, F.W., "A Planform Parameter for Correlating Certain Aerodynamic Characteristics of Swept Wings," NACA TN 2335, 1951.

5 Hauptman, A., and Miloh, T., "On the Exact Solution of the Linearized Lifting-Surface Problem of an Elliptic Wing," Quarterly Journal of Mechanics and Applied Mathematics, Vol. 39, Feb. 1986, pp. 41-66.

6 Laitone, E.V., "Lift-Curve Slope for Finte-Aspect-Ratio Wings," Journal of Aircraft, Vol. 26, No. 8, Aug. 1989, pp. 789-790.

7 Sobieszczanski-Sobieski, J., "A System Approach to Aircraft Optimization," AGARD Rept. 784, Feb. 1992, pp. 2-1-2-15.

8 Livne, E., "Analytic Sensitivities for Shape Optimization in Equivalent Plate Structural Wing Models," Submitted for publication to Journal of Aircraft, 1993.

9 Rudisill, C.S., and Bhatia, K.G., "Optimization of Complex Structures to Satisfy Flutter Requirements," AIAA Journal. Vol. 9, No. 8, August 1971, pp. 1486-1491. 
10 Pedersen, P., and Seyranian, A.P., "Sensitivity Analysis for Problems of Dynamic Stability," International Journal of Solids and Structures, Vol. 19, No. 4, 1983, pp. 315-335.

11 Hawk, D.J., and Bristow, D.R., "Development of MCAERO Wing Design Panel Method with Interactive Graphics Module," NASA CR-3775, 1984.

12 Barthelemy, J.-F.M., and Bergen, F.D., "Shape Sensitivity Analysis of Wing Static Aeroelastic Characteristics," NASA TP-2808, May 1988.

13 Kapania, R.K., "Sensitivity Analysis of Dynamic Aeroelastic Responses," AGARD Rept. 784, Feb. 1992, pp. 3-1-3-12.

14 Kapania, R.K., Bergen, F.D., and Barthelemy, J.-F.M., "Shape Sensitivity Analysis of Flutter Response of a Laminated Wing," AIAA Journal, Vol. 29, No. 4, 1991, pp. 611612. Also presented at 30th AIAA/ASME/ASCE/AHS/ASC Structures, Structural Dynamics and Materials Conference, Mobile, Alabama, 1989 and NASA-CR-181725.

15 Kida, T., and Miyai, Y., "An Alternative Treatment of Lifting-Line Theory as a Perturbation Problem," ZAMP, Vol. 29, 1978, pp. 591-607.

16 Helmbold, H.B., "Der Unverwundene Ellipsenflügel als Tragende Fläche," Jahrbuch 1942 der dutscher Luftfahrtforschung, 10 Lieferung, pp. 2-4.

17 Issac, J.C., and Kapania, R.K., "Sensitivity Analysis of Dynamic Aeroelastic Responses in Transonic Flow," Department of Aerospace Engineering, Virginia Polytechnic Institute and State University, Blacksburg, VA, 1993.

18 Desmarais, R.N., and Bennet, R.M., "User's Guide for a Modular Flutter Analysis Software System," NASA TM 78720, May 1978. 
Table 1. Flutter speed of the wing at $\mathrm{M}=\mathbf{0 . 9}$

$\left(\right.$ Area $=20 \mathrm{~m}^{2}$, Aspect ratio $=10$, Taper ratio $\left.=0.5\right)$

\begin{tabular}{|c|c|c|c|c|}
\hline \multirow{2}{*}{$\begin{array}{c}\text { Sweep } \\
\text { angle }(\mathrm{deg})\end{array}$} & $2 \mathrm{D}$ & Correction 1 & Correction 2 & Correction 3 \\
\cline { 2 - 5 } & 229.90 & 242.96 & 242.94 & 242.07 \\
15 & 209.67 & 234.50 & 234.49 & 233.71 \\
30 & 213.22 & 265.61 & 265.59 & 264.74 \\
\hline
\end{tabular}

Table 2. Sensitivity of flutter speed of the wing at $M=0.9$

$\left(\right.$ Area $=20 \mathrm{~m}^{2}$, Aspect ratio $=10$, Taper ratio $\left.=0.5\right)$

with respect to shape parameters for different $C_{N_{\alpha}}$ corrections

\begin{tabular}{|c|c|c|c|c|c|}
\hline \multirow{2}{*}{$\begin{array}{c}\text { Sweep } \\
\text { angle }(\mathrm{deg})\end{array}$} & Parameter & \multicolumn{4}{|c|}{ Analytic flutter speed derivative } \\
\cline { 3 - 6 } & & $2 \mathrm{D}$ & Correction 1 & Correction 2 & Correction 3 \\
\hline \multirow{2}{*}{ Aspect ratio } & -12.6812 & -14.8836 & -14.8772 & -13.9977 \\
& Area & -6.4607 & -6.8354 & -6.8349 & -6.8101 \\
& Taper ratio & -184.8167 & -196.6132 & -196.5961 & -195.7870 \\
& Sweep angle & -104.4327 & -114.8496 & -114.833 & -114.0609 \\
\hline \multirow{2}{*}{15} & Aspect ratio & -10.1067 & -12.7430 & -12.7374 & -12.0213 \\
& Area & -4.3196 & -4.7405 & -4.7402 & -4.7281 \\
& Taper ratio & -199.1987 & -228.2302 & -228.2101 & -227.2566 \\
& Sweep angle & -37.7435 & 46.6035 & 46.6031 & 46.5800 \\
\hline 30 & Aspect ratio & -6.6440 & -10.1090 & -10.1031 & -9.4892 \\
& Area & -5.0568 & -6.1086 & -6.1082 & -6.0919 \\
& Taper ratio & -172.6965 & -226.3212 & -226.3015 & -225.3706 \\
& Sweep angle & 67.8415 & 191.7299 & 191.7178 & 191.1474 \\
\hline
\end{tabular}



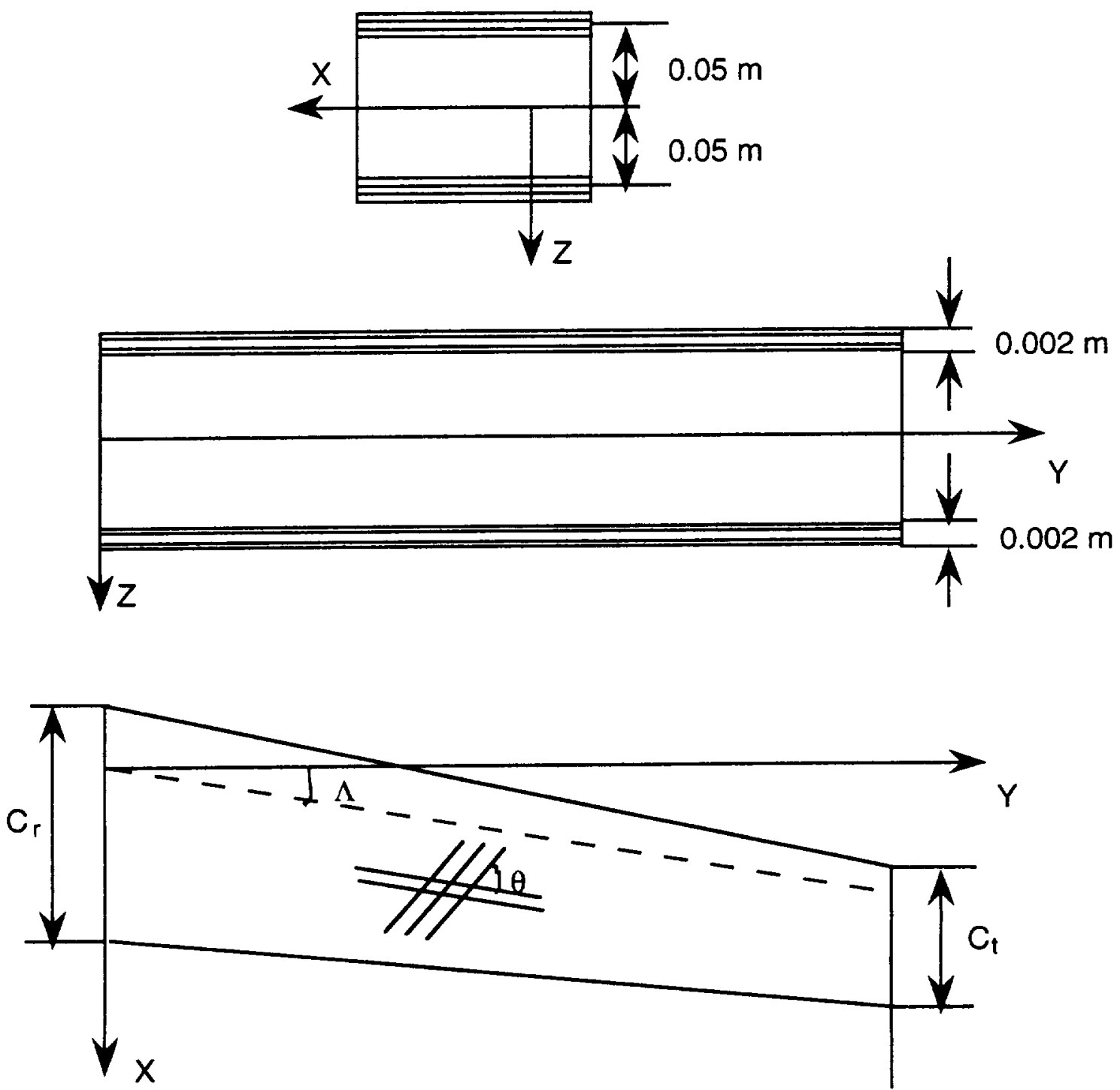

Fig 1. Wing section used 


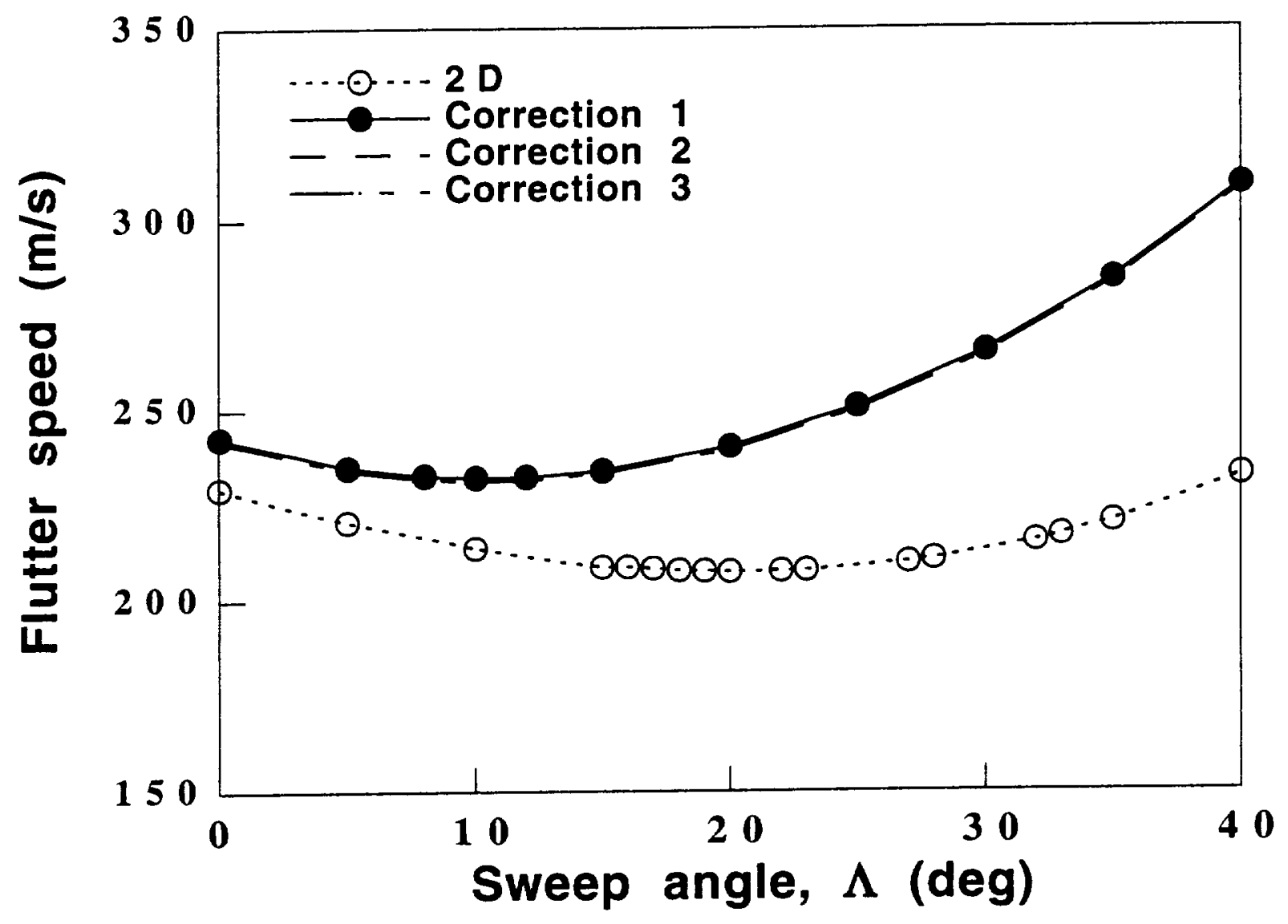

Fig 2. Flutter speed Vs Sweep Angle ( $A R=10$, Area $=20 \mathrm{~m}^{2}, T R=0.5$ ) 


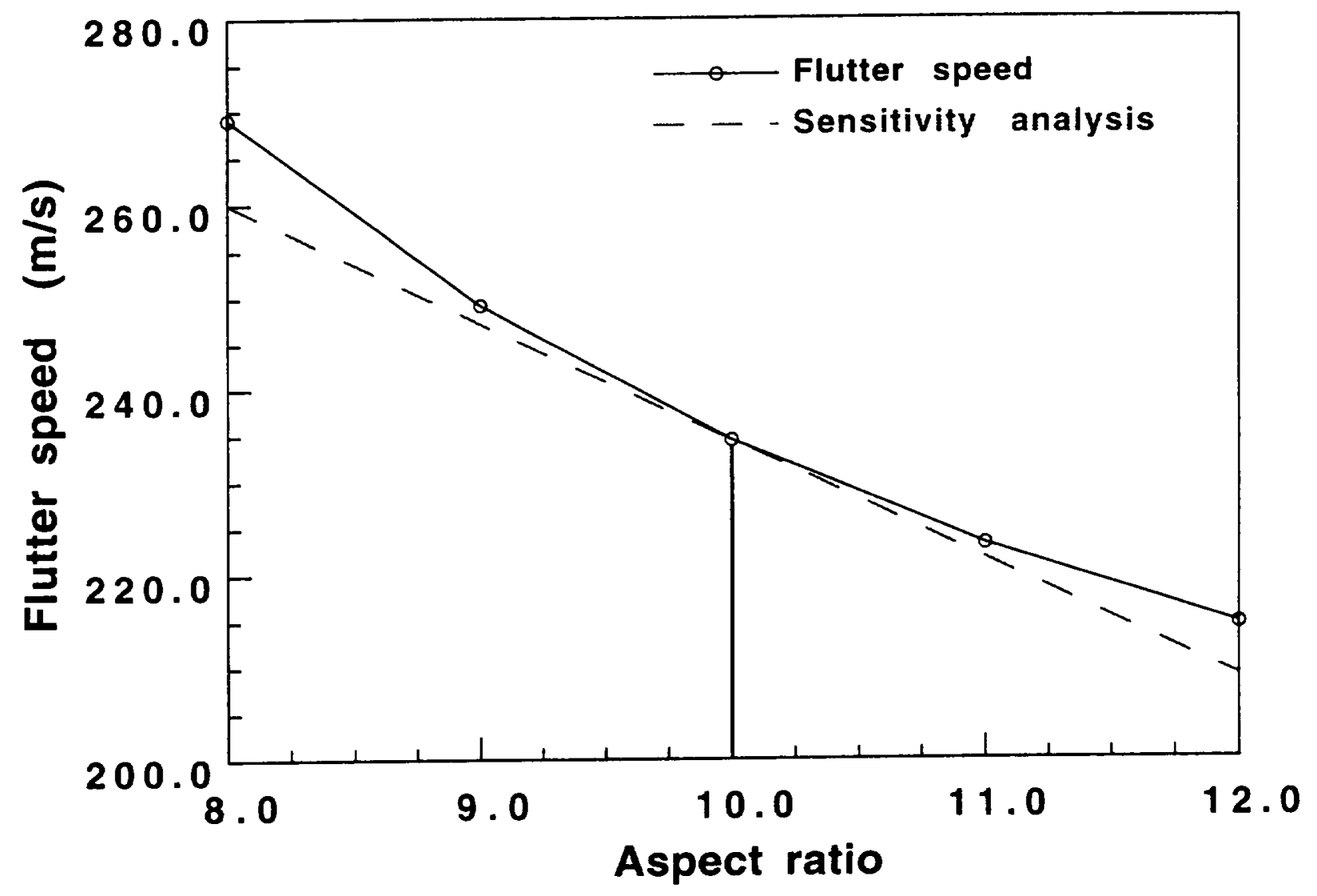

Fig 3. Flutter speed Vs Aspect ratio $(\mathbf{M}=\mathbf{0 . 9})$

$\left(A R=10\right.$, Area $=20 \mathrm{~m}^{2}, T R=0.5$, Sweep $\left.=15^{\circ}\right)$ with finite-span corrections (Correction 1) 


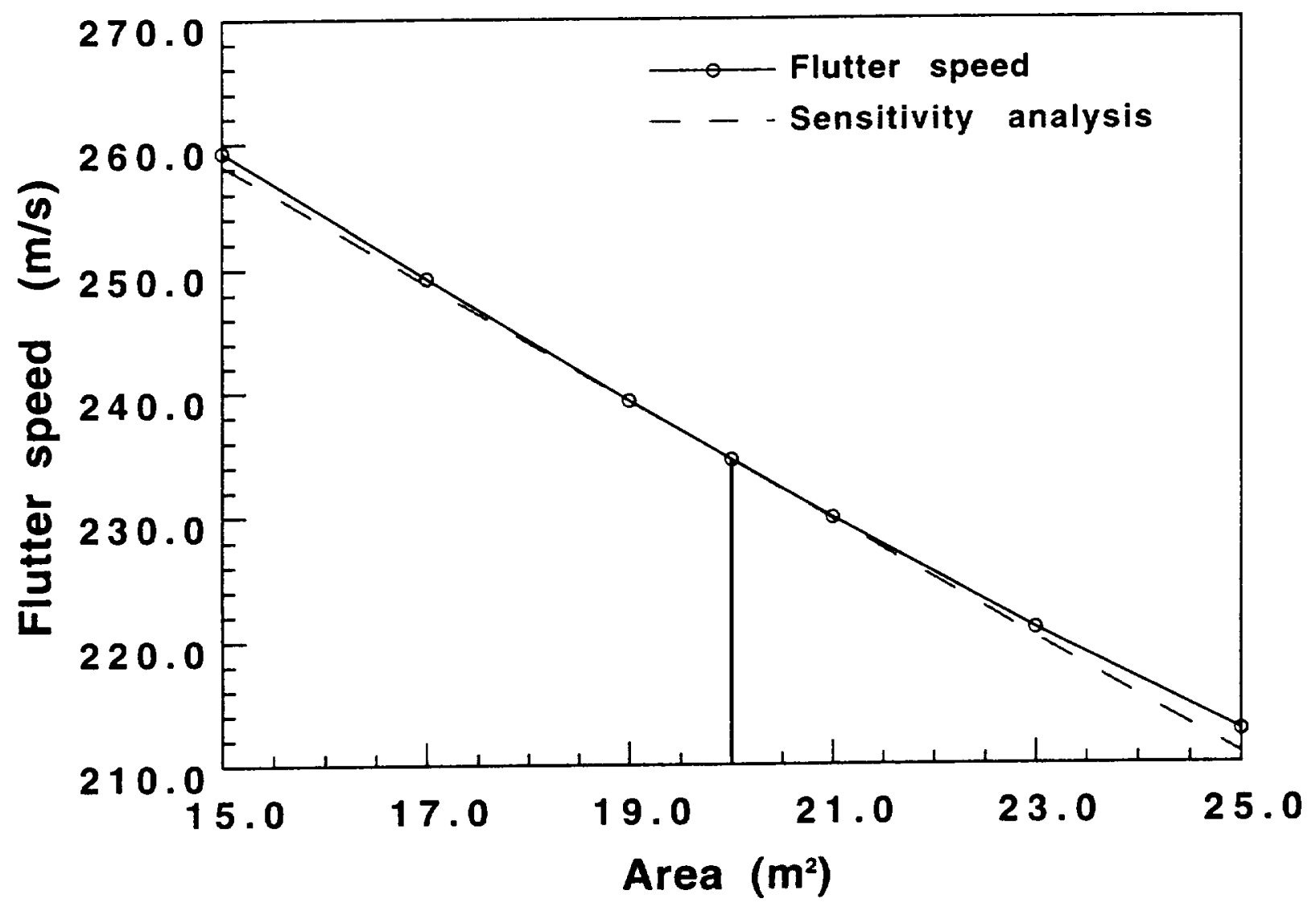

Fig 4. Flutter speed Vs Area $(M=0.9)$

$$
\left(A R=10, \text { Area }=20 \mathrm{~m}^{2}, T R=0.5 \text {, Sweep }=15^{\circ}\right)
$$
with finite-span corrections (Correction 2) 


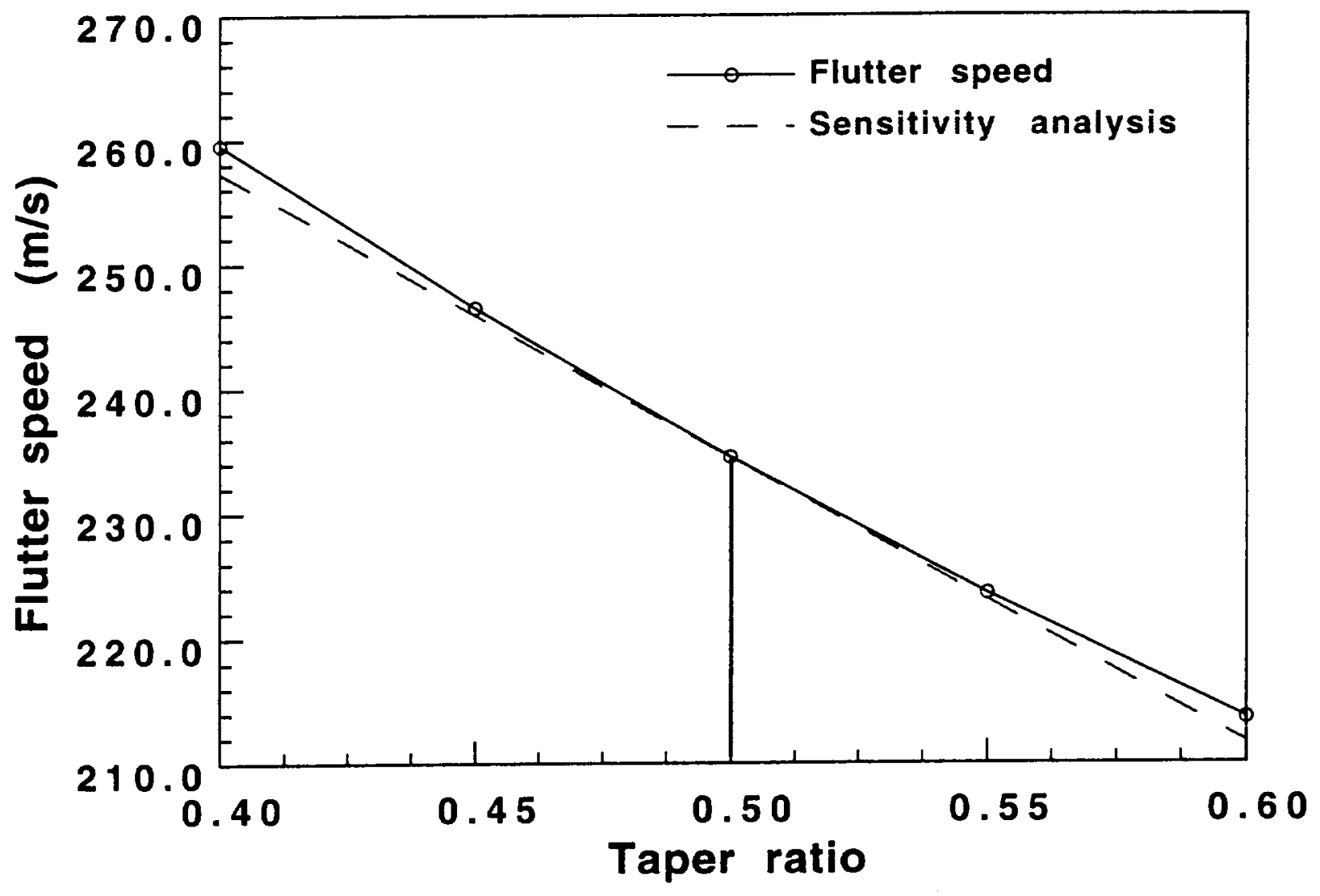

Fig 5. Flutter speed Vs Taper ratio $(\mathbf{M}=\mathbf{0 . 9})$

(AR=10, Area $=20 \mathrm{~m}^{2}, T R=0.5$, Sweep $=15^{\circ}$ ) with finite-span corrections (Correction 3) 


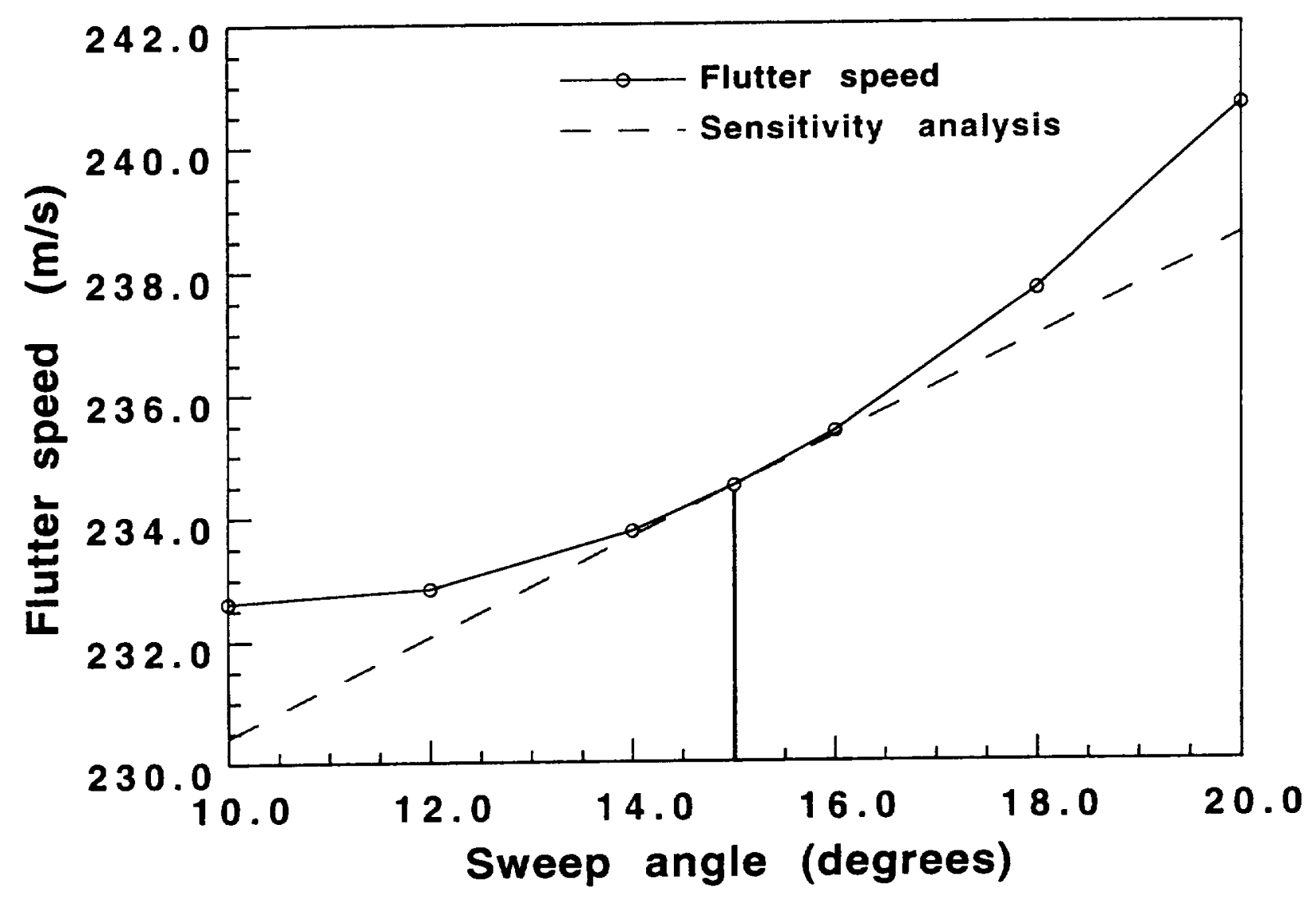

Fig 6. Flutter speed Vs Sweep angle $(M=0.9)$

$\left(A R=10\right.$, Area $=20 \mathrm{~m}^{2}, T R=0.5$, Sweep $=15^{\circ}$ ) with finite-span corrections (Correction 1) 